\title{
Opinion
}

\section{Preparing for an In vitro Fertilization Cycle}

\author{
Connie Stark, RNC, CPC* \\ ART of Wellness Certified Nurse Specialist and Fertility Consultant, Chicago, Illinois, USA \\ "Corresponding authors \\ Connie Stark, RNC, CPC \\ Founder and President of ART of Wellness, ART of Wellness Certified Nurse Specialist and Fertility Consultant, Chicago, Illinois, USA; \\ E-mail: artofwellnesscoach@gmail.com
}

\section{Article information}

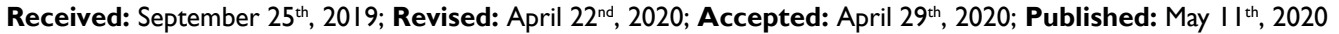

\section{Cite this article}

Stark C. Preparing for an in vitro fertilization cycle. Women Health Open J. 2020; 6(I): 8-9. doi: 10.17140/WHOJ-6-137

$\mathrm{P}$ ursuing the path to parenthood is a time of life that we should never take for granted. There are many paths to parenthood. For some people, the only way of achieving pregnancy is pursuing an in vitro fertilization (IVF) cycle.

This path can be filled with a mix of emotions that can be overwhelming and stressful creating feelings of fear of the unknown. Patients who seek emotional support early in treatment are often better prepared for their experiences and find it significantly less stressful than patients who do not, according to Dr. Ali Domar, founder of mind/body programs for women's health".

There are ways to prepare for an IVF cycle that can aid in managing these emotions making this an exciting time while enhancing positive outcomes. Education of the IVF process and understanding the procedures, self-care and nurturing support systems are key action steps when making the IVF decision.

\section{EDUCATION AND UNDERSTANDING OFTHE IVF PLAN AND PROCESS}

Prior to meeting with your physician and starting your IVF cycle, please follow the below steps:

- Read through the materials provided by your clinic.

- Write down all questions prior to your appointment you may have, bring to your appointment.

- Bring along your schedule for work, vacation and personal dates so you can coordinate the treatment with the clinic. what to expect from your appointments?

- The first appointment with the doctor usually covers the required pre-testing prior to treatment, procedures, medications, complications, protocols statistics and your personal possible outcomes.
- A second meeting may be with the IVF coordinator who will educate you on the treatment plan, review and execute consents, order medications, schedule blood and ultrasound appointments

- In the U.S, a meeting with the financial advisor will review possible insurance coverage, authorizations and out of pocket expenses.

\section{SELF CARE}

\section{Prepare to be Your Best Self}

Preparing for an IVF cycle when dealt with an emphasis on self care, the experience will feel more positive and enabling. This is a challenging time. It is extremely important for men and women to practice self-care. Making changes to diet, exercise and managing stress levels are all key, fertility nurse consultant guides patients towards 5 areas to enhance fertility wellness ${ }^{2}$ :

Mind/Mindset: Be aware of your thoughts. Let positive thoughts dominate the negative. Prepare your mind to accept a positive outcome throughout your IVF cycle, do not prepare for the "What if it does not work?"

Physical Self: Proper nutrition, exercise, supplements, and sleep provide a healthy environment and give the best support during the IVF cycle. Nicotine, caffeine and alcohol have negative effects on outcomes.

Emotional Self: Recognize feelings of excitement, love, nervousness, stress, loss, grief and other emotions. When stressed with negative feelings such as fear, sadness, anxiety, the stress hormone cortisol increases which impacts the entire body. Take time to relax and practice stress management techniques, such as - gentle ex- 
ercise, journaling, social activities, naps, relaxation techniques and talking through your emotions.

Social Self: It is difficult to initiate or participate in social activities, especially if babies and children are included. This is natural and understandable. Social activities can help your emotions, de-stress, lift your mood or act as a distraction. ${ }^{3}$

Financial Self: The financial side of treatments can be overwhelming. Plan and work out finances beforehand and perhaps a new job, additional insurance, extra savings.

\section{SUPPORT SYSTEMS}

During the fertility journey support systems are very important. Leaning on family and friends to support social and interpersonal needs. Doctors and nurses educate and guide throughout the process and offer advice and support. Personalized fertility coaching focuses on support and strategies that enhance preconception health. ${ }^{4}$ Choosing a coach with fertility expertise ensures guided support to focus on all 5 points to enhance wellness and a journey that feels like you're never alone. Joining an in person or online support group is another option that may validate and support personal experiences. The coach should have fertility expertise to ensure you have the guided support needed to never feel alone during the fertility journey.
It is believed and witnessed that "Success happens for those who are willing to make every day changes and embrace every opportunity to enhance their fertility. Understanding how the mind and body respond to a temporary crisis like infertility and having the ability to fully prepare for the next step impacts positive outcomes." 5

\section{REFERENCES}

1. Domar A. Domar Center for Mind/Body Health. domarcernter Web site. https://www.domarcenter.com/about/staff/alice_ domar. Accessed September 24, 2019.

2. Domar A. Mind/body guide to enhancing fertility. In: Conquering-Infertility. London, United Kingdom: Penguin Books; 2004.

3. RESOLVE The National Infertility Association. resolve Web site. https://resolve.org/. Accessed September 24, 2019.

4. American Society for Reproductive Medicine (ASRM). reproductivefacts Web site. https://www.reproductivefacts.org/. Accessed September 24, 2019.

5. Cwikel J, Gidron Y, Sheiner E. Psychological interactions with infertility among women. Eur J Obstet Gynecol Reprod Biol. 2004; 117(2): 126-131. doi: 10.1016/j.ejogrb.2004.05.004 